\title{
Multilevel Homotopic Adaptive Finite Element Methods for Convection Dominated Problems ${ }^{\star}$
}

Long Chen, Pengtao Sun, and Jinchao Xu

The Pennsylvania State University, Department of Mathematics.

Summary. A multilevel homotopic adaptive finite element method is presented in this paper for convection dominated problems. By the homotopic method with respect to the diffusion parameter, the grids are iteratively adapted to better approximate the solution. Some new theoretic results and practical techniques for the grid adaptation are presented. Numerical experiments show that a standard finite element scheme based on this properly adapted grid works in a robust and efficient manner.

\section{Introduction}

In this paper, we shall present a class of adaptive finite element methods (FEMs) for the convection-dominated problems. One simple model is the following convection-diffusion problem:

$$
-\epsilon \Delta u+b(x) \cdot \nabla u=f(x),
$$

which is posed on a bounded domain $\Omega \subset R^{2}$ with a proper boundary condition.

We are interested in adaptive finite element methods for the convectiondominated case, namely $\epsilon$ is sufficiently smaller than $b(x)$. It is well-known that one major difficult for this type of problem is that a standard finite element discretization scheme usually fail and specialized methods, such as upwinding scheme and streamline diffusion methods, need to be adapted. One conclusion from the study in this paper is that a standard finite element scheme still works reasonably well if the grid is properly adapted so that sharp boundary or internal layers presented in the solution will be fully resolved. To obtain such a properly adapted grid, we are going to use a homotopic

\footnotetext{
* This work was supported in part by NSF DMS-0074299, NSF DMS-0209497,NSF DMS-0215392 and the Center for Computational Mathematics and Application at Penn State.
} 
method with respect to the diffusion parameter $\epsilon$. Namely, we first start our computation for large $\epsilon$, say $\epsilon=1$ and use adaptive grid technique for elliptic problems to obtain a good initial grid. We then start to decrease the value of $\epsilon$ and use the current grid as an initial grid to obtain a new adaptive grid. We continue in this way until the desired value of $\epsilon$ is reached. As a general approach, the homotopic method of this type is commonly used in many different application areas and there have been existing works for grid adaptation (c.f. Habashi et al. [1997]). What is of importance here is how such a continuation procedure is carried out in a robust and efficient manner. In this paper, we will first present an interpolation error estimate and then develop local mesh improvement techniques such as refinement, coarsening and smoothing and global moving mesh strategy which aims to minimize the interpolation error.

\section{Theoretical foundation}

In this section, we include an interpolation error estimate from Chen et al. [2003]. (Similar error estimates in special cases can also be found in, e.g, D'Azevedo and Simpson [1989], Habashi et al. [1997], Huang and Sun [2003]). This estimate can be viewed as the theoretical foundation of this paper, namely our algorithms are aimed at minimizing (or at least reducing) this interpolation error by iteratively modifying our grids.

The estimate.

Let $\Omega$ be a bounded domain in $R^{n}$. Given a function $u \in \mathcal{C}^{2}(\bar{\Omega})$, we call a symmetric positive definite matrix $H \in R^{n \times n}$ to be a majorizing Hessian of $u$ if

$$
\left|\xi^{t}\left(\nabla^{2} u\right)(x) \xi\right| \leq c_{0} \xi^{t} H(x) \xi, \quad \xi \in R^{n}, x \in \Omega
$$

for some positive constant $c_{0}$.

We then use the majorizing Hessian to define a new metric

$$
H_{p}=(\operatorname{det} H)^{-\frac{1}{2 p+n}} H, p \geq 1
$$

There are two conditions for a triangulation $\mathcal{T}_{N}$, where $N$ is the number of simplexes, to be a nearly optimal mesh in the sense of minimizing the interpolation error in $L^{p}$ norm. The first assumption asks the mesh to capture the high oscillation of the Hessian metric, namely $H$ does not change very much on each element.

(A1) There exist two positive constants $\alpha_{0}$ and $\alpha_{1}$ such that

$$
\alpha_{0} \xi^{t} H_{\tau} \xi \leq \xi^{t} H(x) \xi \leq \alpha_{1} \xi^{t} H_{\tau} \xi, \quad \xi \in R^{n},
$$

where $H_{\tau}$ is the average of $H$ over $\tau$. 
The second condition asks that $\mathcal{T}_{N}$ is quasi-uniform under the new metric induced by $H_{p}$.

(A2) There exists two positive constants $\beta_{0}$ and $\beta_{1}$ such that

$$
\frac{\sum_{i} \tilde{d}_{\tau, i}^{2}}{|\tilde{\tau}|^{2 / n}} \leq \beta_{0}, \forall \tau \in \mathcal{T}_{N} \text { and } \frac{\max _{\tau \in \mathcal{T}}|\tilde{\tau}|}{\min _{\tau \in \mathcal{T}}|\tilde{\tau}|} \leq \beta_{1}
$$

where $|\tilde{\tau}|$ is the volume of $\tau$ and $\tilde{d}_{\tau, i}$ is the length of the $i$-th edge of $\tau$ under the new metric $H_{p}$, respectively.

The first inequality in (2) means that each $\tau$ is isotropic i.e. shape-regular under the metric $H_{p}$. The second inequality (2) means that all elements $\tau$ are of comparable size (under the new metric), which is a global condition and known as the equidistribution principal Huang [2001]. It means that the mesh will concentrate at the region where $\operatorname{det} H_{p}(\mathbf{x})$ is large. We proved in Chen et al. [2003] that a triangulation which satisfies both local and global conditions yields a good approximation.

Theorem 1. Let $u \in \mathcal{C}^{2}(\bar{\Omega}), \mathcal{T}_{N}$ satisfy assumptions (A1) and (A2) and $u_{I}$ is the linear finite element interpolation of $u$ based on the triangulation $\mathcal{T}_{N}$, the following error estimate holds:

$$
\left\|u-u_{I}\right\|_{L^{p}(\Omega)} \leq C N^{-2 / n}\|\sqrt[n]{\operatorname{det}(H)}\|_{L^{\frac{p n}{2 p+n}}(\Omega)}
$$

for some constant $C=C\left(n, p, c_{0}, \alpha_{0}, \alpha_{1}, \beta_{0}, \beta_{1}\right)$. This error estimate is optimal in the sense that for a strictly convex (or concave) function, the above inequality holds in a reversed direction.

It is well known that for diffusion dominated problems, there will be some sharp boundary layers or internal layers with width in $\epsilon$ scale. We would like to emphasis that the error bound in Theorem 1 is independent of $\epsilon$. Some numerical results about the $\epsilon$ independence of the interpolation error can be found at Chen et al. [2003].

As mentioned before, Theorem 1 will be the basis of grid adaptation algorithms. Roughly speaking, for a given function $u$, we will adapt our grids in such a way the assumption (A1) and (A2) will be better and better satisfied. One important remark we need to make is that the validity of Theorem 1 allows for a few exceptions of the assumption (A2) for $p<\infty$, see Chen et al. [2003] for details. This is particularly important since in practice it is very difficult to guarantee that (A2) is satisfied everywhere. We note that the theory and algorithms in D'Azevedo and Simpson [1989] and Habashi et al. [1997] are only for $p=\infty$ which requires that (A2) be satisfied on each element in the triangulation.

Postprocessing: recovery of Hessian.

In this section, we will discuss how the Hessian matrix of the solution can be obtained when linear finite element approximation is used for the discretization of partial differential equations. Since taking piecewise second derivatives 
to piecewise linear functions will given no approximation to Hessian matrix, special Postprocessing techniques need to be used to obtain reasonable Hessian matrix approximation from linear finite elements.

One most popular technique is a patch recovery technique proposed by Zienkiewicz and J.Z.Zhu [1992a,b] which is based on the least squares fitting locally. Results from their application demonstrate that it is robust and efficiency. The theoretical reason for ZZ method to work is largely understood to be related to the superconvergence phenomenon for second order elliptic boundary value problems discretized on a finite element grid that has certain local symmetry, see Wahlbin [1995], Chen and Huang [1995], Babuska and Strouboulis [2001]. These classic superconvergence results can be used to justify the effectiveness of Zienkiewicz-Zhu method, see, for example, Zhang [1999], Li and Zhang [1999] for nearly structured grids. A significant improvement of this type of analysis was given recently by Bank and Xu [2003a,b]. In Bank and $\mathrm{Xu}$ [2003a] they gave superconvergence estimates for piecewise linear finite element approximation on quasi-uniform triangular meshes where most pairs of triangles sharing a common edge form approximate parallelograms. They also analyze a postprocessing gradient recovery scheme, showing that $Q_{h} \nabla u_{h}$ is a superconvergent approximation to $\nabla u$. Here $Q_{h}$ is the global $L^{2}$ projection. This result leads to a theoretical justification of $\mathrm{ZZ}$ method for such type of grids, see Xu and Zhang [2004]. Recently, Carstensen and Bartels [2002] also gave theoretical and numerical support for the robust reliability of all averaging techniques on unstructured grids.

The gradient recovery algorithm used in the numerical examples of this paper is based on a new approach due to Bank and Xu [2003b] where they use the smoothing iteration of the multigrid method to develop a postprocessing gradient recovery scheme. This scheme proves to be very efficient for recovering Hessian matrix. All the above methods can be extended to anisotropic grids with some proper modifications, but a theoretical justification of such extensions is still lacking. Nevertheless, numerical experiments have given satisfactory results.

\section{Mesh adaptation}

In this section, we will discuss techniques which aim at improving the mesh quality. Here we define the mesh quality for a triangulation $\mathcal{T}$ by the interpolation error:

$$
Q(\mathcal{T}, u, p)=\left\|u-u_{I, \mathcal{T}}\right\|_{L^{p}(\Omega)}, 1 \leq p \leq \infty .
$$

Local mesh optimizations.

There are mainly three types mesh improvements: (1) refinement or coarsening Bank et al. [1983], Rivara [1984], Kornhuber and Roitzsch [1990], (2) edge swapping Lawson [1977], and (3) mesh smoothing Bank and Smith [1997], 
Jones et al. [1995]. We will derive those techniques by minimizing the interpolation error in $L^{p}$ norm, which can be achieved by equidistributing edge lengths under the new metric.

We compute edge lengths under the new metric $H_{p}$ and mark edges whose lengths are greater than $r_{1} d$, where $r_{1} \geq 1$ is a parameter and $d$ is a fixed edge length. We connect marked edges element-wise according to different situations; See Fig. 1.

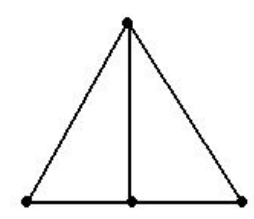

1 marked edge

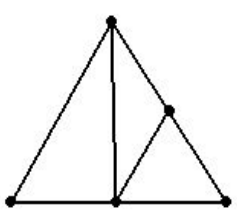

2 marked edges

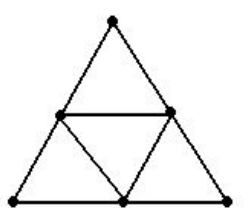

3 marked edges

Fig. 1. Edge-based refinement

The coarsening operates like an inverse procedure of refinement. It marks the one whose length is less than $r_{2} d$ for another parameter $r_{2} \leq 1$. We then shrink this edge to a point and connect to the vertices of the patch of the edge.

Now we consider the edge swapping for four points $\left\{\mathbf{v}_{i}\right\}_{i=1}^{4}$ which form two adjacent triangles and a convex quadrilateral. Let $\mathcal{T}_{1}=\triangle_{123} \cup \triangle_{134}$ and $\mathcal{T}_{2}=\triangle_{124} \cup \triangle_{234}$, where $\triangle_{i j k}$ stands for the triangle made up by $\mathbf{v}_{i}, \mathbf{v}_{j}$, and $\mathbf{v}_{k}$. We choose triangulation $\mathcal{T}_{1}$ if and only if $Q\left(\mathcal{T}_{1}, u, p\right) \leq Q\left(\mathcal{T}_{2}, u, p\right)$, for some $1 \leq p \leq \infty$. In Chen and $\mathrm{Xu}$ [2004a], we show this criteria is equivalent to the empty circle criteria when $u(\mathbf{x})=\|\mathbf{x}\|^{2}$. Thus it is an appropriate generalization of the edge swapping used in the isotropic case to the anisotropic case.

Local mesh smoothing adjusts the location of a vertex in its patch $\Omega_{i}$, which consists of all simplexes containing vertex $\mathbf{x}_{i}$, without changing the connectivity. Moving vertex to the new location will provably or heuristically improve the mesh quality. Several sweeps through the whole mesh can be performed to improve the overall mesh quality. By minimizing the interpolation error in $\Omega_{i}$, we move $\mathbf{x}_{i}$ to $\mathbf{x}^{*}$ such that

$$
\nabla u\left(\mathbf{x}^{*}\right)=-\frac{1}{\left|\Omega_{i}\right|} \sum_{\tau_{j} \in \Omega_{i}}\left(\nabla\left|\tau_{j}\right| \sum_{\mathbf{x}_{k} \in \tau_{j}, \mathbf{x}_{k} \neq \mathbf{x}_{i}} u\left(\mathbf{x}_{k}\right)\right) .
$$

The derivation of this formula can be found at Chen and $\mathrm{Xu}$ [2004a]. In the application to numerical solution, we use $Q_{h} \nabla u_{h}$ and $u_{h}$ in (3) to perform the calculation. 
Global moving mesh strategy.

Another global approach to improve the mesh to better approximate a solution has been carried out in the study of the so-called moving mesh method Huang [2001], Huang and Sun [2003], Huang and Russell [1999]. Let $\Omega^{c}$ be the computational domain with a quasi-uniform (under the standard Euclidean metric) triangulation $T_{N}^{c}$. The mesh on $\Omega$ can be viewed as the image of a transformation $x=x(\xi): \Omega^{c} \rightarrow \Omega$. Then to ask the transformed mesh to be quasi-uniform respect to the metric $G(x)$ is more or less equivalent to ask $x=x(\xi)$ to be the global minimizer of the minimizing problem:

$$
\min _{x} \int_{\Omega^{c}}\left(\sum_{i}\left(\nabla x_{i}\right)^{t} G(x) \nabla x_{i}\right)^{q} d \xi, q>n / 2 .
$$

The minimizers of the above functionals is expected to satisfy both equidistribution and isotropy conditions simultaneously Chen et al. [2003]. We note that the $q=1$ case corresponds to the harmonic mapping but we ask $q>n / 2$ here. When $n \geq 3$, these minimization problems (which is more or less $p$-Laplacian with $p>n$ ) is significantly different from the harmonic mapping which has been most commonly used in the literature for moving mesh method Liseikin [1999], Dvinsky [1991]. If we choose $G=[\operatorname{det}(H)]^{-1 /(2 p+n)} H$ in the functional, we can get a nearly optimal mesh which minimizes the interpolation error $\left\|u-u_{I}\right\|_{L^{p}(\Omega)}$ by solving above functional.

\section{Numerical examples}

Our multilevel homotopic adaptive grid method for convection dominated problem $-\epsilon \Delta u+b(x) \cdot \nabla u=f(x)$ can be roughly described as follows.

Given $\rho=\epsilon_{0} \gg \epsilon$ and $h=h_{0}$, generate an initial mesh $\mathcal{T}_{h}$.

1. Discretize the PDE on mesh $\mathcal{T}_{h}$ and solve it to get the solution $u_{h}$.

2. Global or local move $\mathcal{T}_{h}$ using $u_{h}$ and its recovered derivatives.

3. If $\rho=\epsilon$, locally improve the grid using the estimated new metric. Otherwise go to (4).

4. Global refine $\mathcal{T}_{h}$, and set $\rho \leftarrow \gamma \rho(\gamma<1), h \leftarrow h / 2$. Go to (1).

Let's considering the following convection dominated model problem:

$$
\left\{\begin{aligned}
-\epsilon \Delta u+u_{x} & =1 x \in \Omega \\
u & =0 x \in \partial \bar{\Omega}
\end{aligned}\right.
$$

on the unit square domain $\Omega=(0,1)^{2}$ with $\epsilon=0.001$. By applying above algorithm we solve this convection-diffusion problem and try to catch out the singular sections of solution by means of moving mesh and local optimized mesh. The following pictures in Fig. 2 describe that how the adaptive mesh 

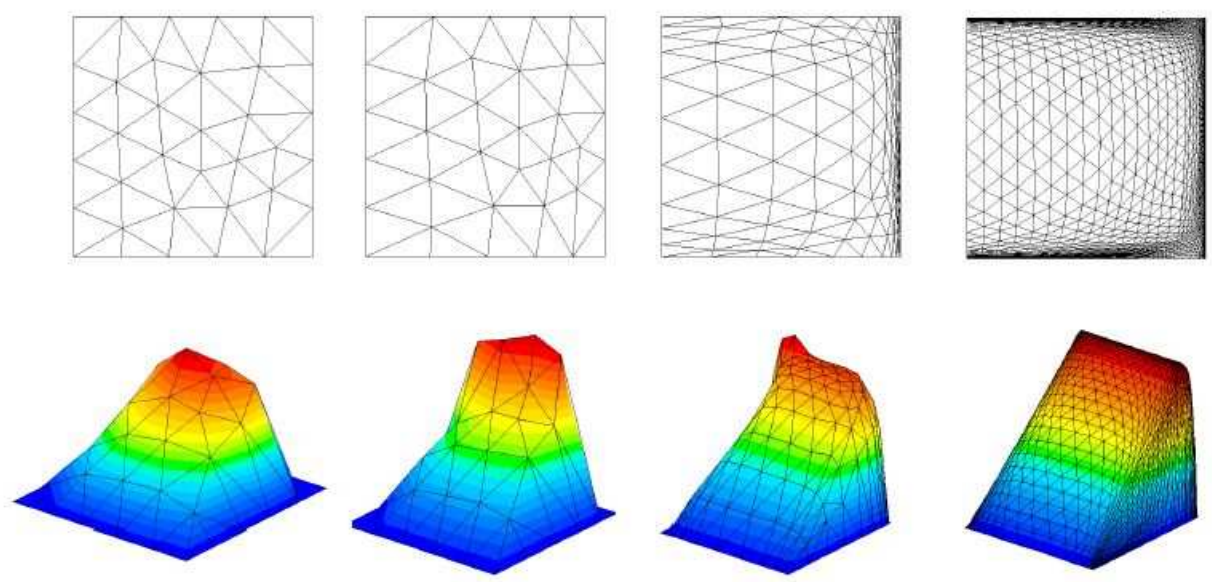

Fig. 2. Continuation adaptive meshes and corresponding solutions

and numerical solution change in this multilevel homotopic adaptive process step by step.

In this process of multilevel homotopic adaptive mesh, we start from a very coarse initial grid with respect to $\epsilon=0.1$. On the following each level, we decrease $\epsilon$ once by dividing by 2 firstly, then resolve the original P.D.E (4) on this level's mesh with the standard finite element method, and get more significant improvements of numerical solution $u_{h}$ around the upper, lower and right boundary layer. After that, we calculate the modified Hessian matrix $H_{p}$ (in this example, we choose $p=1$ i.e. we measure the error in $L^{1}$ norm) of the solution via the recovery technique we mentioned in section 3. Then according to the value of Hessian matrix on each mesh element, we move grids to upper, lower and right boundary layer by virtue of moving mesh method. On the other hand, when we decrease $\epsilon$, we apply global mesh refinement for the whole domain in order to get more grids to move.

Keep running this process until $\epsilon$ equals to its original value 0.001 , we then begin to do the local mesh optimizations. Still utilizing the Hessian matrix $H_{p}$ of each mesh element, we catch and mark those edges whose lengths under the new matrix is relative large, then apply our local refinement technique on these marked edges and thus locally generate a finer mesh to resolve the singularity of the solution.

Eventually the singularity of the solution of (4) is resolved, no oscillation any more. To show the numerical optimal convergence rate of our algorithm, we list the error in $L^{1}$ norm and its convergence rate in Table 1.

Since the analytical solution to (4) is not available, we compute a solution on a very fine Shishkin mesh Shishkin [1990] for which the near optimal convergence result is known Roos [2002] and use it as the real solution to compute the error. We apply our algorithm with different initial meshes to obtain a 


\begin{tabular}{c|c|c}
\hline$N$ & error & Rate \\
\hline 4712 & $2.400665 \mathrm{E}-04$ & 0.99 \\
7043 & $9.871067 \mathrm{E}-05$ & 1.04 \\
10102 & $9.144469 \mathrm{E}-05$ & 1.01 \\
14329 & $7.822302 \mathrm{E}-05$ & 0.99 \\
17929 & $9.253636 \mathrm{E}-05$ & 0.95 \\
22256 & $6.092786 \mathrm{E}-05$ & 0.97 \\
\hline
\end{tabular}

Table 1. Errors of FEM on adapted grids

sequence of near optimal meshes for the linear interpolant. The first column of Table 1 is the number of nodes (unknowns) and the second one is the $L^{1}$ norm of the error. In the third column, we list the ratio $\ln$ error $/ \ln N$. It is clear that the standard finite element on the nearly optimal meshes obtain an optimal convergence rate.

\section{Concluding remarks}

In this paper, we have shown an optimal interpolation error estimates in $L^{p}$ norm and, based on the estimate, we have developed new techniques, including local mesh optimizations and global moving mesh strategy, to improve the mesh to better approximate the solution. Those techniques with the homotopy with respect to diffusion coefficient are successfully applied to convection dominated problem. One main observation in our work is that a properly adapted mesh will enhance the stability of the standard finite element methods which often fails for convection dominated problems on quasi-uniform grids. This phenomenon has been observed in other simpler situations (see books Miller et al. [1996], Roos et al. [1996]). In the current work, the mesh is adapted to optimize the interpolation error. We expect that the discretization error will inherit the optimality of the interpolation error on a nearly optimal mesh for linear interpolant. We have obtained some preliminary results for a 1-d convection dominated model problem Chen and Xu [2004b] which shows that the optimality of the convergence rate is sensitive to the perturbation of meshes in the smooth part.

Another critical question that needs to be addressed is that how to solve these sequences of systems efficiently since in the adaptive procedure described in this paper, we need to solve many systems of algebraic system of equations. Hence how to solve these sequence of systems efficiently is crucial to the entire adaptive procedure. It is in fact the main research interest of the authors to develop efficient methods for such systems. We need to develop techniques how to make use of the intermediate grids and equations together with their discrete solutions. This is a subject of our ongoing research. 


\section{References}

I. Babuska and T. Strouboulis. The finite element method and its reliability. Numerical Mathematics and Scientific Computation. Oxford Science Publications, 2001.

R. E. Bank, A. H. Sherman, and A. Weiser. Refinement algorithms and data structures for regular local mesh refinement. In R. S. et al., editor, Scientific Computing, pages 3-17. IMACS/North-Holland Publishing Company, Amsterdam, 1983.

R. E. Bank and R. K. Smith. Mesh smoothing using a posteriori error estimates. SIAM Journal on Numerical Analysis, 34:979-997, 1997.

R. E. Bank and J. Xu. Asymptotically exact a posteriori error estimators, Part I: Grids with superconvergence. SIAM J. on Numerical Analysis, 41 (6):2294-2312, 2003a.

R. E. Bank and J. Xu. Asymptotically exact a posteriori error estimators, Part II: General unstructured grids. SIAM J. on Numerical Analysis, 41 (6):2313-2332, 2003b.

C. Carstensen and S. Bartels. Each averaging technique yields reliable a posteriori error control in FEM on unstructured grids. I. low order conforming, nonconforming, and mixed FEM. Math. Comp., 71(239):945-969, 2002.

C. Chen and Y. Huang. High accuracy theory of finite element methods. Hunan, Science Press, Hunan, China (in Chinese), 1995.

L. Chen, P. Sun, and J. Xu. Optimal anisotropic simplicial meshes for minimizing interpolation errors in $L^{p}$-norm. Submitted to Math. Comp., 2003.

L. Chen and J. Xu. Optimal Delaunay triangulation. J. of Comp. Math., 22 (2):299-308, 2004a.

L. Chen and J. Xu. Stability and accuracy of adapted finite element methods for singularly perturbed problems. Technical report, 2004b.

E. D'Azevedo and R. Simpson. On optimal interpolation triangle incidences. SIAM Journal on Scientific and Statistical Computing, 6:1063-1075, 1989.

A. S. Dvinsky. Adaptive grid generation from harmonic maps on riemannian manifolds. J. Comput. Phys., 95(2):450-476, 1991.

W. Habashi, M. Fortin, J. Dompierre, M. Vallet, D. A. A. Yahia, Y. Bourgault, M. Robichaud, A. Tam, and S. Boivin. Anisotropic mesh optimization for structured and unstructured meshes. In 28th Computational Fluid Dynamics Lecture Series 1997-02. von Karman Institute for Fluid Dynamics, 1997.

W. Huang. Variational mesh adaptation: isotropy and equidistribution. J. Comput. Phys., 174:903-924, 2001.

W. Huang and R. D. Russell. Moving mesh strategy based on a gradient flow equation for two-dimensional problems. SIAMJournal on Scientific Computing, 20(3):998-1015, 1999.

W. Huang and W. Sun. Variational mesh adaptation II: Error estimates and monitor functions. J. Comput. Phys., 2003. 
M. Jones, L. Freitag, and P. Plassmann. An efficient parallel algorithm for mesh smoothing. In 4th Int. Meshing Roundtables, pages 47-58. Sandia Labs, 1995.

R. Kornhuber and R. Roitzsch. On adaptive grid refinement in the presence of internal or boundary layers. IMPACT Comput. Sci. Engrg., 2:40-72, 1990.

C. Lawson. Software for $C^{1}$ surface interpolation. In J. Rice, editor, Mathematical Software III, pages 161-194. Academic Press, 1977.

B. Li and Z. Zhang. Analysis of a class of superconvergence patch recovery techniques for linear and bilinear finite elements. Numerical Methods for Partial Differential Equations, pages 151-167, 1999.

V. Liseikin. Grid Generation Methods. Springer Verlag, Berlin, 1999.

J. Miller, E. O'Riordan, and G. Shishkin. Fitted Numerical Methods For Singular Perturbation Problems. World Scientific, 1996.

M. Rivara. Mesh refinement processes based on the generalized bisection of simplexes. SIAM J. Numer. Anal., 21:604-613, 1984.

H. Roos. Optimal convergence of basic schemes for elliptic boundary value problems with strong parabolic layers. J. Math. Anal. Appl., 267:194208, 2002.

H. Roos, M. Stynes, and L. Tobiska. Numerical Methods for Singularly Perturbed Differential Equations, volume 24 of Springer Series in Computational Mathematics. Springer Verlag, 1996.

G. Shishkin. Grid approximation of singularly perturbed elliptic and parabolic equations (in Russian). PhD thesis, Second doctorial thesis, Keldysh Institute, Moscow, 1990.

L. Wahlbin. Superconvergence in Galkerkin finite element methods. Springer Verlag, Berlin, 1995.

J. $\mathrm{Xu}$ and $\mathrm{Z}$. Zhang. Analysis of recovery type a posteriori error estimators for mildly structured grids. Math. Comp., 73 (247):1139-1152, 2004. URL http://www.ams.org/mcom/2004-73-247/ S0025-5718-03-01600-4/home.html.

Z. Zhang. Ultraconvergence of the patch recovery technique II. Mathematics Of Computation, 69(229):141-158, 1999.

O. Zienkiewicz and J.Z.Zhu. The superconvergence patch recovery and a posteriori error estimates. Part 1: The recovery techniques. Int. J. Number. Methods Engrg., 33:1331-1364, 1992a.

O. Zienkiewicz and J.Z.Zhu. The superconvergence patch recovery and a posteriori error estimates. Part 2: Error estimates and adaptivity. Int. J. Number. Methods Engrg., 33:1365-1382, 1992b. 